\title{
Experimental Validation and Application of a Phased Array Ultrasonic Testing Model on Sound Field Optimization
}

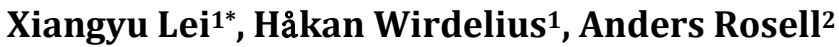 \\ ${ }^{1}$ Department of Industrial and Materials Science, Chalmers University of Technology, Gothenburg, Sweden \\ ${ }^{2}$ GKN Aerospace Sweden AB, Flygmotorvägen 1, Trollhättan, Sweden \\ Email: *xlei@chalmers.se
}

How to cite this paper: Lei, X., Wirdelius, H. and Rosell, A. (2021) Experimental Validation and Application of a Phased Array Ultrasonic Testing Model on Sound Field Optimization. Journal of Modern Physics, 12, 391-407.

https://doi.org/10.4236/jmp.2021.124028

Received: February 1, 2021

Accepted: February 28, 2021

Published: March 3, 2021

Copyright $\odot 2021$ by author(s) and Scientific Research Publishing Inc. This work is licensed under the Creative Commons Attribution International License (CC BY 4.0).

http://creativecommons.org/licenses/by/4.0/ (c) (i) Open Access

\begin{abstract}
In safety dominant industries, nondestructive evaluation (NDE) is crucial in quality assurance and assessment. Phased array ultrasonic testing (PAUT) as one of the NDE methods is more promising compared with conventional ultrasonic testing (UT) method in terms of inspection speed and flexibility. To incorporate PAUT, the techniques should be qualified, which traditionally is performed by extensive physical experiments. However, with the development of numerical models simulating UT method, it is expected to complement or partly replace the experiments with the intention to reduce costs and operational uncertainties. The models should be validated to ensure its consistency to reality. This validation work can be done by comparing the model with other validated models or corresponding experiments. The purpose of current work focuses on the experimental validation of a numerical model, simSUNDT, developed by the Chalmers University of Technology. Validation is conducted by comparing different data presentations (A-, B- and C-scan) from experimental and simulated results with some well-defined artificial defects. Satisfactory correlations can be observed from the comparisons. After the validation, sound field optimization work aiming at retrieving maximized echo amplitude on a certain defect can be started using the model. This also reveals the flexibility of parametric studies using simulation models.
\end{abstract}

\section{Keywords}

Experiments, Simulation, Validation, Sound Field Optimization

\section{Introduction}

In safety prioritized industries such as aerospace industry, newly adopted and 
advanced technologies of Additive Manufacturing (AM) enable innovative designs of complex-shaped components and have the potential to broaden the manufacturing possibilities. Since the safety aspect is crucial and must not be compromised, these new manufacturing approaches demand even higher accuracy and reliability in quality assessments. Nondestructive evaluation (NDE) is used to ensure the quality and integrity of the manufactured components and among others, conventional ultrasonic testing (UT) has been widely used in industries [1]. Within this area, the phased array ultrasonic testing (PAUT) technique has many possible advantages over conventional single-element UT methods in terms of operational flexibility and increased inspection speed [2]. However, the complexity and flexibility of PAUT raise the need of understanding the technique in qualification and evaluation procedures. There is also a need of adjustment for AM-specific defect characteristics and geometrical constraints of specific parts.

The traditional approach to qualification work is associated with extensive and expensive physical experiments. However, with the development of mathematical modelling of NDE methods in the last decades, the experimental work can be supported or partly replaced by the model-based data, provided that the model itself has been validated [3]. The validation of the mathematical model can be done by comparing it with other already validated models, but it should ultimately be compared with physical experiments in all perspectives to ensure its accuracy. Some works had been devoted to experimental validation of a simulation model in terms of PAUT, see for example [4] and [5].

The phased array (PA) probe model implemented in a UT simulation software, simSUNDT, developed by the Chalmers University of Technology, had been validated to some extent in terms of maximum echo amplitude towards well-defined artificial defects, i.e. side-drilled holes (SDHs) [6]. In the current paper, the model is to be further validated by comparing the data presentations (A-, B- and C-scan). These are retrieved from both experiments and corresponding simulations, which address some well-defined artificial defects in noise-free test specimens. Satisfactory correlations can be observed from the comparisons and the model can be concluded as an alternative to the corresponding experiments. The generated sound fields towards a certain type of defect, i.e. surface breaking crack, are optimized with the help of this validated model as an applied practice. The optimization in this work aims at retrieving a maximized echo amplitude by adjusting the combination of probe angle and focusing distance, which is easy and essential to change in the phased array configuration. The possibility and procedure of using the simulation model in sound field optimization work is hereby investigated.

\section{2. simSUNDT Software}

The simSUNDT software, developed at Chalmers University of Technology, composes of a Windows-based processor for simulation definition and result 
analysis. A mathematical kernel, UTDefect, which conducts the actual mathematical modelling [7] [8] [9] [10], has been validated by comparing with previous done and in literature available experiments [7] [8] [9] [11]. The 3D elastodynamic wave equation that defines the wave propagation in a homogeneous half space is solved by using vector wave functions [10]. The modelled geometry can be described as a plate bounded by the scanning surface, on which rectangular mesh is used to define the scanning sequence. Scattering by defects is solved using analytical or semi-analytical methods.

The simSUNDT can simulate the entire NDE inspection. In order to accomplish this, calibration option with reference reflector is available. These reflectors include side-drilled hole (SDH) [12] and flat-bottom hole (FBH), which is represented by a cylindrical cavity and an open circular crack, respectively.

The volumetric and crack-like defects are available types of defect to be simulated. The volumetric ones include a spherical/spheroid cavity (pore), a spherical inclusion (isotropic material differing from the surrounding material, i.e. slag) and a cylindrical cavity (SDH). Crack-like ones include rectangular/circular crack (lack of fusion) and strip-like crack (fatigue crack). Tilting planar back surface could also be modelled for the strip-like crack, but it is otherwise assumed parallel to the scanning surface. The surface-breaking strip-like crack and rectangular crack close to the back surface can be used to model the corresponding defects in the test piece.

The conventional contact probe is represented as the boundary conditions representing a plane wave in the far-field at a certain angle. Different traction conditions represent different probe types under the effective area and are assumed to be zero elsewhere. This enables the possibilities of simulating any types of the probe available on the market, by specifying related parameters such as wave types, crystal size and shape, angles, frequency ranges, contact conditions, etc. In addition, it is also possible to suppress the mode-converted wave component in the simulation to eventually facilitate the analysis of the received signal. By modelling the receiver, a reciprocity argument [13] is applied. The arrangement of the probe can be chosen among pulse-echo, separate with fixed transmitter and tandem configuration (TOFD).

Above principles are the same for the PA probe model, i.e. the individual element is represented by the corresponding boundary conditions. The individual boundary conditions are translated into the main coordinate system and a PA wave front with certain nominal angle is formulated by constructive phase interference.

\section{Experiments}

\subsection{Experimental Instruments and Test Specimens}

The data acquisition hardware TOPAZ64 is a portable 64-channel PAUT device. It is connected to a computer with data communication through Gigabyte Ethernet cable. Operations are monitored on software UltraVision, which pro- 
vides real-time data presentation and visualization. The linear PA probe connected to TOPAZ64 is labelled as LM-5MHz, which contains 64 elements that generate longitudinal waves. As indicated by its nomination, the nominal center frequency is $5 \mathrm{MHz}$ and bandwidth (-6 dB) is $74 \%$. Table 1 lists some key specifications of this probe. In addition, two plastic wedges with and without angle labelled as LM-55SW and LM-0LW, respectively, are incorporated in all experiments to protect the probe surface and to facilitate fixation of the probe on the mechanized gantry system. This gantry system is motor-controlled in horizontal $(\mathrm{x}-\mathrm{y})$ plane while the vertical position (z-axis) is manually adjusted at this moment. The bottom end of the $\mathrm{z}$-axis has a spring load fork that can clamp the wedge with probe. The motors are embedded with encoders so that the horizontal position of the probe can be obtained and read by UltraVision to present position-related visualizations.

There are two stainless-steel test specimens with different artificial defects (SDH and surface breaking crack) included in this work, see Figure 1. The first specimen (\#1) has $6 \mathrm{SDHs}$ ( $2 \mathrm{~mm}$ in diameter) at depth from $10 \mathrm{~mm}$ to $60 \mathrm{~mm}$ with increment of $10 \mathrm{~mm}$. The second one (\#2) has 5 vertical surface breaking cracks (tilt angle of 0 -degree) with height of $0.5 \mathrm{~mm}, 2 \mathrm{~mm}, 5 \mathrm{~mm}, 10 \mathrm{~mm}$ and $15 \mathrm{~mm}$. Table 2 summarizes the overall dimension and acoustic properties of

Table 1. Specification of the linear PA probe used in the experiment.

\begin{tabular}{cc}
\hline Specification & Value \\
\hline Total elements & 64 \\
Total length $(\mathrm{mm})$ & 38.3 \\
Elevation $(\mathrm{mm})$ & 10 \\
Pitch $(\mathrm{mm})$ & 0.6 \\
Kerf & 0.1 \\
Center frequency $(\mathrm{MHz})$ & 5 \\
Relative bandwidth (\%) & 74
\end{tabular}

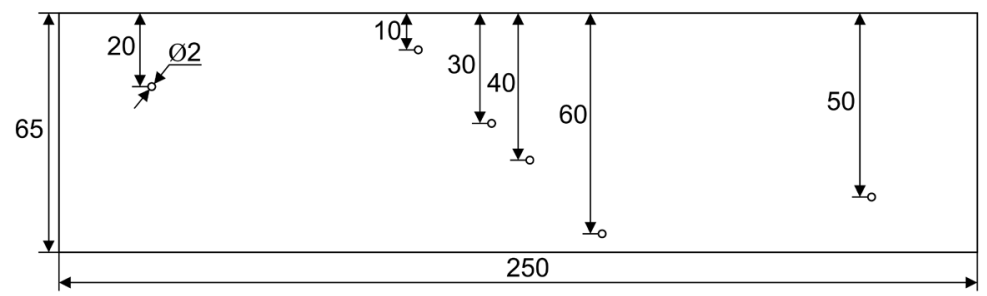

(a) Test specimen \#1 with SDHs at different depth

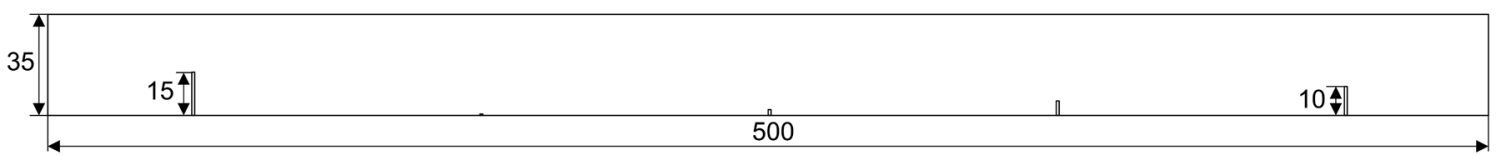

(b) Test specimen \#2 with cracks, height from left to right is $15 \mathrm{~mm}, 0.5 \mathrm{~mm}$ (too small to be seen in the figure), $2 \mathrm{~mm}, 5 \mathrm{~mm}$. $10 \mathrm{~mm}$

Figure 1. Sketch profiles of the test specimens. 
Table 2. Dimensions and acoustic properties of specimens.

\begin{tabular}{cccccc}
\hline No. & $\begin{array}{c}\text { Length } \\
(\mathrm{mm})\end{array}$ & $\begin{array}{c}\text { Height } \\
(\mathrm{mm})\end{array}$ & $\begin{array}{c}\text { Width } \\
(\mathrm{mm})\end{array}$ & $\begin{array}{c}\text { L-wave speed } \\
(\mathrm{m} / \mathrm{s})\end{array}$ & $\begin{array}{c}\text { T-wave speed } \\
(\mathrm{m} / \mathrm{s})\end{array}$ \\
\hline$\# 1$ & 250 & 65 & 39 & 5640 & 3110 \\
$\# 2$ & 500 & 35 & 50 & 5573 & 3150 \\
\hline
\end{tabular}

these specimens and all these defects span through the entire width of each specimen.

\subsection{Experimental Configurations}

The validations presented in the current work aim at comparing different data presentations (A-scan from the maximum echo amplitude, B- and C-scans) between physical experiments and corresponding simulations. Data acquisition is performed in pulse-echo mode and the probe inspects a test specimen with one-line scan on the specimen surface to obtain the signals from all involved defects at once.

On the first test specimen (\#1), only the SDH at $50 \mathrm{~mm}$ depth is studied under four inspection cases, i.e. (a) non-angled sound beam without focusing effect; (b) non-angled sound beam with focusing at $50 \mathrm{~mm}$ depth; (c) 45-degree angled sound beam without focusing effect and (d) 45-degree angled sound beam with focusing at $50 \mathrm{~mm}$ depth. The direct echo from the $\mathrm{SDH}$ is stored as received signal.

On the second test specimen (\#2), all cracks except the largest one (height of $15 \mathrm{~mm}$ ) are studied under two general inspection cases, i.e. (a) 45-degree angled sound beam without focusing effect and (b) 45-degree angled sound beam with focusing at $35 \mathrm{~mm}$ depth. The surface breaking cracks are positioned so that the openings are on the bottom surface and corner echoes are the received signals.

Within all these inspection cases, only the central 16 elements are activated in non-focusing cases to prevent ghost images, whereas all 64 elements are activated to generate proper focusing effect.

\section{Simulation Configuration}

To compare with experimental data presentations, the corresponding one-line scan simulations must cover a certain scan interval. This is to ensure that the maximum echo amplitude from the defect of interest can be retrieved to be presented in A-scan, and that sufficient amount of scan positions can be covered to visualize the defect in B-scan and echo dynamic curve in C-scan. Furthermore, the sampling time steps of A-scan signals should be small enough to ensure the accuracy of detailed comparisons. Under above reasons, all simulations for validation purpose are conducted in large scan and time intervals with scan increment of $0.1 \mathrm{~mm}$ and time step of $0.005 \mu$ s. These simulation parameters were determined in such a way that the resulted data presentations have a decent resolution to be compared with experiments. Parameters related to the PA probe 
are set in accordance with the physical probe as in Table 1.

As expected, the simulation time is positively correlated with the amount of scan and time positions, as well as the complexity of studied defect and inspection scenario. The simulation times of cracks with height of $5 \mathrm{~mm}$ and $10 \mathrm{~mm}$ involving focusing effect were observed to be extensively long using above mentioned simulation configurations. To avoid this, only certain scan and time intervals where the maximum echo amplitudes are expected were simulated for these two cracks with focusing effect, while still keeping the scan increment of $0.1 \mathrm{~mm}$ and time step of $0.005 \mu$ s unchanged. For this reason, the B-scan comparisons are neglected for these two cases due to insufficient scan positions.

\section{Probe Skew Angle Sensitivity}

For the physical experiments, there could be some practical uncertainties in the system affecting the results, one example is the probe skew angle. In order to explore if the minor variation of the probe skew angle can influence the received data, some parametric studies were performed with a set of probe skew angles, ranging from $-5^{\circ}$ to $5^{\circ}$ with an increment of $0.5^{\circ}$. The simulations were performed towards the SDH at $50 \mathrm{~mm}$ depth in test specimen \#1 under the inspection case of 45-degree angled beam with $50 \mathrm{~mm}$ focus depth, just as the case (d) on test specimen \#1 in the experimental configurations. These simulated results are compared with available experimental data.

\section{Optimization of Received Signal}

It is noted that the optimization of the received signal is equivalent to the sound field in this pulse-echo situation. With the help of this simulation model and its flexibility of parametric studies, the generated sound field from a PA probe is explored to be optimized in order to receive a maximized echo amplitude towards a crack, which has a specific character, i.e. size and tilt angle. The considered decision variables to this optimization problem are the probe (beam) angle and focus distance in this work.

The optimization algorithm considered in this work is heuristic Nelder-Mead based Simplex method [14] [15] that solves non-linear single-objective optimization problems. This method compares the objective values at each chosen simplex vertex and moves the next simplex vertex accordingly based on the result evaluations using different operation strategies, i.e. reflection, expansion, contraction and shrink.

The optimization procedure using Simplex algorithm is conducted using software modeFrontier (2020R1), in which the decision variables to an optimization problem are explored. Simulation outputs from simSUNDT can be imported into modeFrontier to help with optimization evolution according to the chosen algorithm.

As mentioned earlier, the simulation towards a large crack where the focusing effect is involved takes extensive simulation time. It is therefore inefficient and 
unfeasible to conduct the actual optimization work based on the simulation configuration (large scan interval, small scan increment, nominal probe bandwidth of $74 \%$, etc.) described in simulation configuration for validation purpose. Based on previous simulation experiences, the simulation time depends positively on the number of involved wave frequencies. Therefore, variations of probe bandwidth are investigated in Table 3 in terms of simulation time and the influence on interested simulation output, i.e. the maximum echo amplitude. The simulated defect is a surface breaking crack (opening at bottom surface) with $10 \mathrm{~mm}$ height and 0-degree tilt angle, corresponds to the $10 \mathrm{~mm}$ height crack in test specimen \#2. Simulation scenario is 45 -degree probe angle and $49.49 \mathrm{~mm}$ focus distance (corresponds to $35 \mathrm{~mm}$ focus depth). The scan interval is chosen so that within which the maximum echo amplitude is expected to appear. Scan positions are uniformly distributed inside this interval.

The maximum echo amplitude of $76.8 \mathrm{~dB}$ in investigation No.1 obtained with nominal probe bandwidth of $74 \%$ is taken as a standard value to upcoming variants (No.2 and 3). Investigation No.2 where bandwidth changes to only $0.02 \%$ gives its maximum echo amplitude of $76.9 \mathrm{~dB}$, which differs only $0.1 \%$ from the standard value but reduces the simulation time significantly up to $91 \%$ if keeping all other parameters unchanged. Moreover, monochromatic frequency spectrum (bandwidth of $0 \%$, only a center frequency content) simulated in investigation No. 3 reduces almost $99 \%$ of standard simulation time, but the maximum echo amplitude of interest changed $8 \%$ to the standard value. These three investigated cases show that the second simulation scheme (bandwidth of $0.02 \%$ ) could be considered reasonable and should be used in the later optimizations.

\section{Results and Discussion}

\subsection{Validity of Simulation Model}

The model is validated in this work by comparing experiments and simulations in terms of data presentations (A-, B- and C-scans), see Figures 2-10, where all signals are normalized to their corresponding maximum values for easy comparison purposes. All simulated results are presented in black colour and experimental ones are in red colour. In A-scans, the experimental peaks (red dotted lines) are shifted in time so that they coincide with simulated peaks (black solid lines) for comparison purpose. The amount of shifted time could be correlated to e.g. experimental condition and setup, etc. In B-scans, the simulated and experimental results are ordered to the left and right, respectively. In C-scans, the

Table 3. Optimization condition investigations.

\begin{tabular}{cccccc}
\hline No. & BW. (\%) & Scan pos. & Time pos. & Max. amp. (dB) & Time (h) \\
\hline 1 & 74 & 35 & 14,448 & 76.8 & 64 \\
2 & 0.02 & 35 & 14,448 & 76.9 & 6 \\
3 & 0 & 35 & - & 83 & 0.13 \\
\hline
\end{tabular}



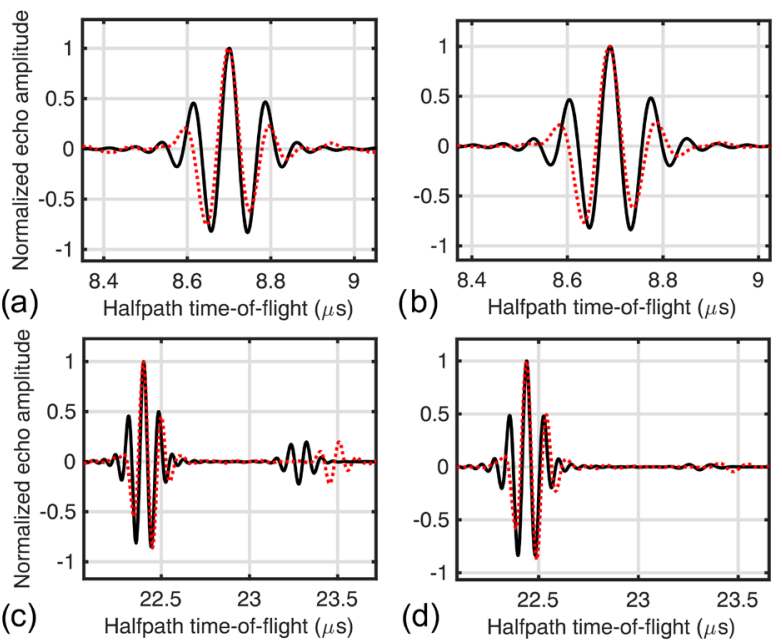

Figure 2. A-scan comparisons between simulations (black solid lines) and experiments (red dotted lines) on SDH at $50 \mathrm{~mm}$ depth in test specimen \#1 under case: (a) non-angled beam without focusing effect, (b) non-angled beam with $50 \mathrm{~mm}$ focus depth, (c) 45-degree angled beam without focusing effect and (d) 45-degree angled beam with 50 $\mathrm{mm}$ focus depth.
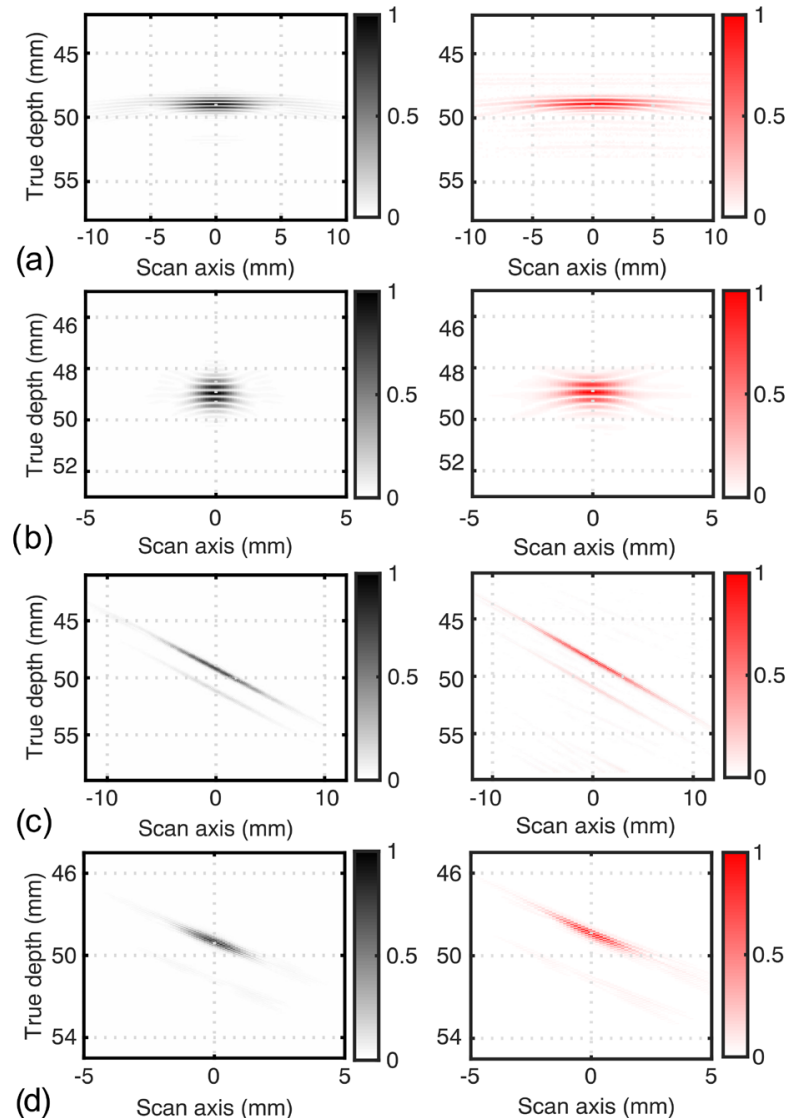

Figure 3. B-scan comparisons between simulations (grey scaled to the left) and experiments (red scaled to the right) on SDH at $50 \mathrm{~mm}$ depth in test specimen \#1 under case: (a) non-angled beam without focusing effect, (b) non-angled beam with $50 \mathrm{~mm}$ focus depth, (c) 45-degree angled beam without focusing effect and (d) 45-degree angled beam with $50 \mathrm{~mm}$ focus depth. 

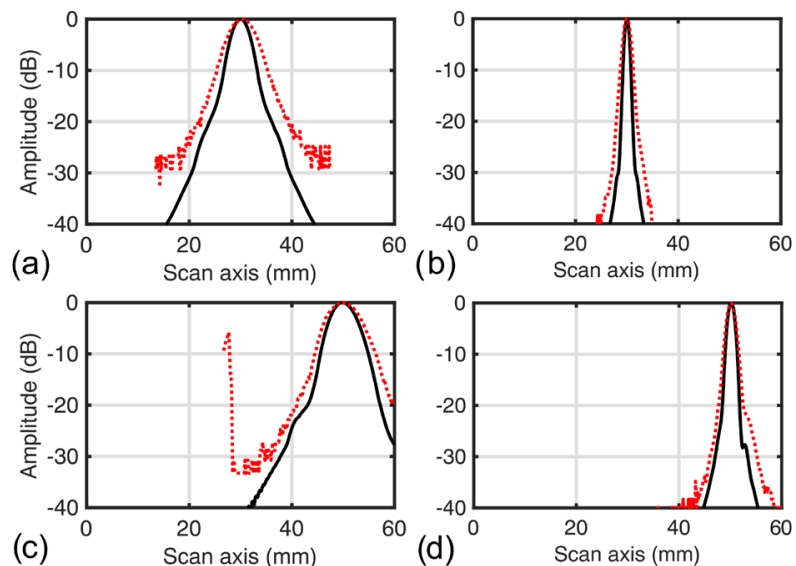

Figure 4. C-scan (echo dynamic curve) comparisons between simulations (black solid lines) and experiments (red dotted lines) on SDH at $50 \mathrm{~mm}$ depth in test specimen \#1 under case: (a) non-angled beam without focusing effect, (b) non-angled beam with 50 $\mathrm{mm}$ focus depth, (c) 45-degree angled beam without focusing effect and (d) 45-degree angled beam with $50 \mathrm{~mm}$ focus depth.

peaks (respective amplitude of $0 \mathrm{~dB}$ corresponds to the scan position where the maximum echo amplitude is detected) are also shifted to facilitate the comparisons.

Comparisons for the SDH at $50 \mathrm{~mm}$ depth in test specimen \#1 are shown in Figures 2-4. The experimental A-scans are shifted by (a) $0.01 \mu \mathrm{s}$, (b) $-0.001 \mu \mathrm{s}$, (c) $0.29 \mu \mathrm{s}$ and (d) $0.2 \mu \mathrm{s}$. Good correlations can be seen in all A- and C-scan comparisons. In one of the A-scans, case (c), a second somewhat weaker pulse can be observed. This is identified as a satellite pulse correlated to the surface wave traveling round the SDH. The mismatch of this weaker wave between simulation and experiment could then be explained by inaccuracy of actual SDH diameter in the test specimen. This weaker pulse in case (d) diminishes since the wave energy is focused and mostly reflected. B-scans show indifferentiable shapes except for the second case, which could be caused by a variation in contact-condition affected beam divergence, but the overall comparisons show good consistency. The echo dynamic curves above $-10 \mathrm{~dB}$ correlate well.

Comparisons for the four surface breaking cracks (height of $0.5 \mathrm{~mm}, 2 \mathrm{~mm}, 5$ $\mathrm{mm}$ and $10 \mathrm{~mm}$ ) in test specimen $\# 2$ without focusing effect are shown in Figures 5-7. The experimental A-scans are shifted by (a) $0.06 \mu \mathrm{s}$, (b) $0.04 \mu \mathrm{s}$, (c) $0.01 \mu$ s and (d) $0.05 \mu \mathrm{s}$. The wave form of the smallest crack (height $0.5 \mathrm{~mm}$ ) does not correlate as good as others and the reason might be that the smallest crack stands more for a volumetric defect than a crack, which was actually modelled and simulated. All B-scans show indifferentiable shapes.

Comparisons for the four surface breaking cracks (height of $0.5 \mathrm{~mm}, 2 \mathrm{~mm}, 5$ $\mathrm{mm}$ and $10 \mathrm{~mm}$ ) in test specimen \#2 with $35 \mathrm{~mm}$ focus depth are shown in Figures 8-10. The experimental A-scans are shifted by (a) $-0.27 \mu$ s, (b) $-0.27 \mu \mathrm{s}$, (c) $-0.14 \mu$ s and (d) $-0.12 \mu \mathrm{s}$. As mentioned in simulation configuration that only a certain scan and time interval where the corresponding maximum echo amplitude is expected was simulated for cracks with height of $5 \mathrm{~mm}$ and $10 \mathrm{~mm}$, their 

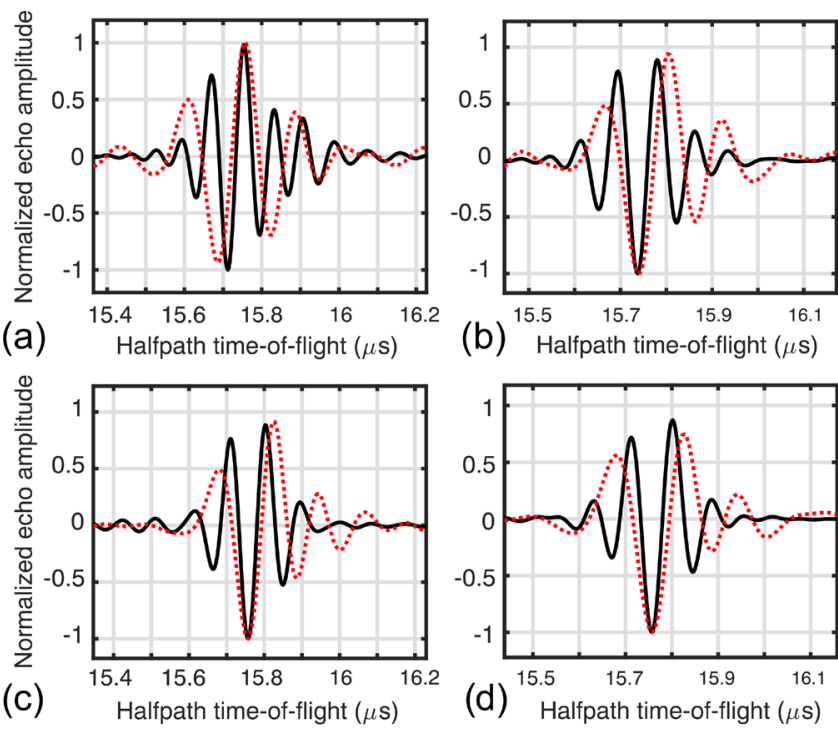

Figure 5. A-scan comparisons between simulations (black solid lines) and experiments (red dotted lines) on different surface breaking cracks in test specimen \#2 without focusing effect, crack height: (a) $0.5 \mathrm{~mm}$, (b) $2 \mathrm{~mm}$, (c) $5 \mathrm{~mm}$, (d) $10 \mathrm{~mm}$.
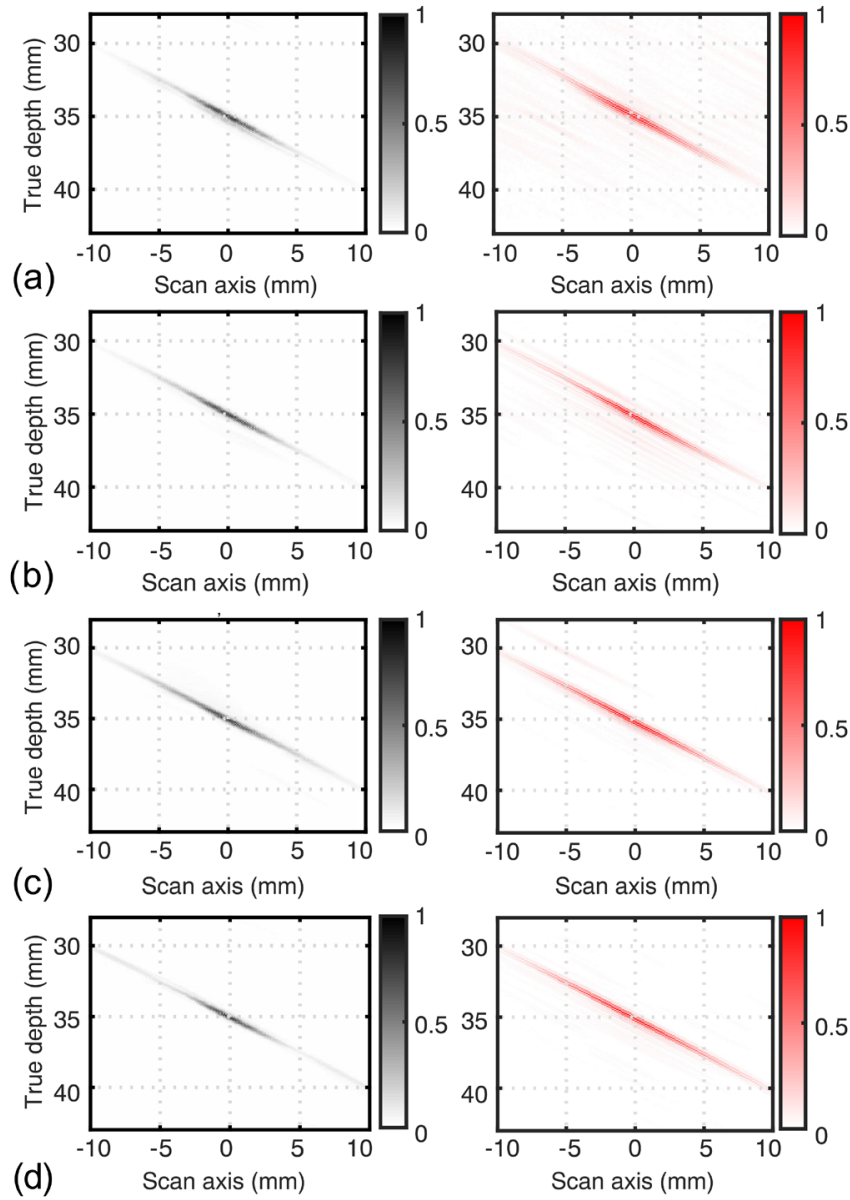

Figure 6. B-scan comparisons between simulations (grey scaled to the left) and experiments (red scaled to the right) on different surface breaking cracks in test specimen \#2 without focusing effect, crack height: (a) $0.5 \mathrm{~mm}$, (b) $2 \mathrm{~mm}$, (c) $5 \mathrm{~mm}$, (d) $10 \mathrm{~mm}$. 


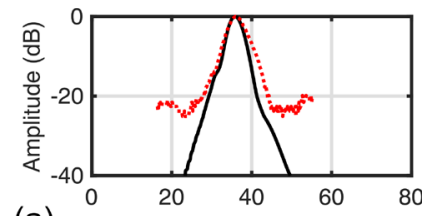

(a)
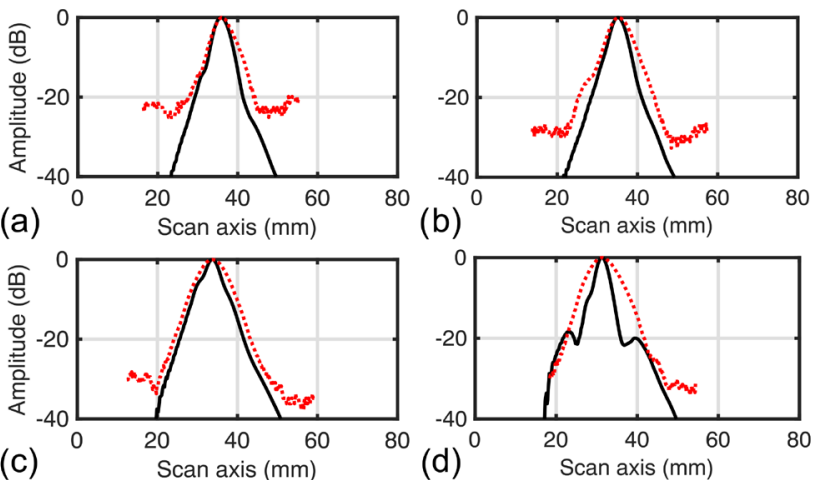

(b)

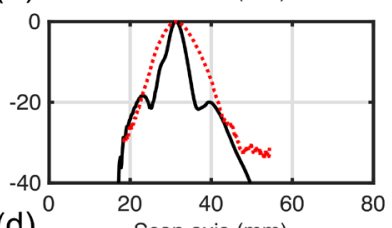

(c)

(d) Scan axis $(\mathrm{mm})$

Figure 7. C-scan (echo dynamic curve) comparisons between simulations (black solid lines) and experiments (red dotted lines) on different surface breaking cracks in test specimen \#2 without focusing effect, crack height: (a) $0.5 \mathrm{~mm}$, (b) $2 \mathrm{~mm}$, (c) $5 \mathrm{~mm}$, (d) $10 \mathrm{~mm}$.
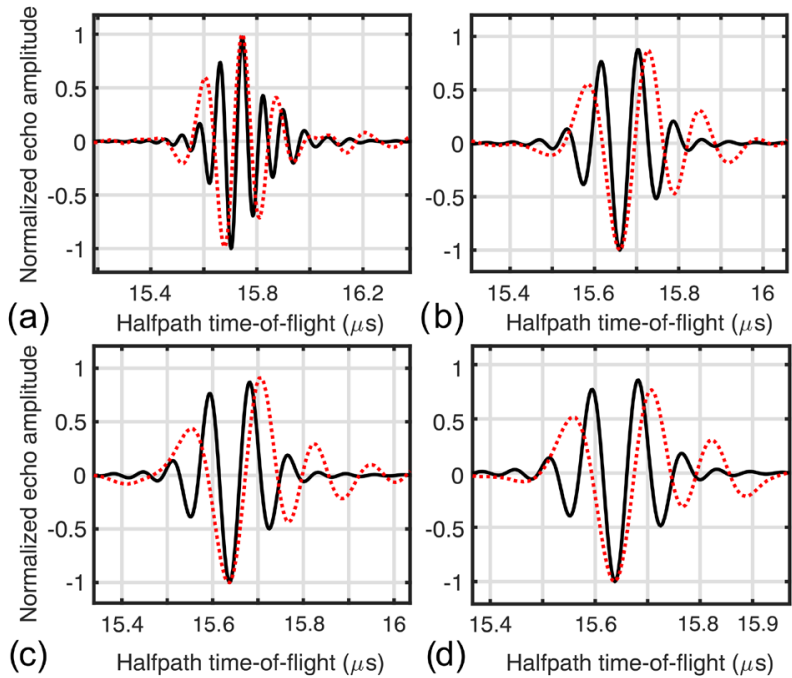

(b) Halfpath time-of-flight $(\mu \mathrm{s})$

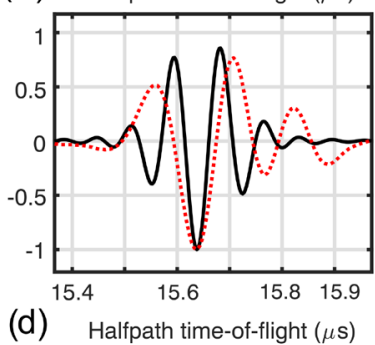

Figure 8. A-scan comparisons between simulations (black solid lines) and experiments (red dotted lines) on different surface breaking cracks in test specimen \#2 with $35 \mathrm{~mm}$ focus depth, crack height: (a) $0.5 \mathrm{~mm}$, (b) $2 \mathrm{~mm}$, (c) $5 \mathrm{~mm}$, (d) $10 \mathrm{~mm}$
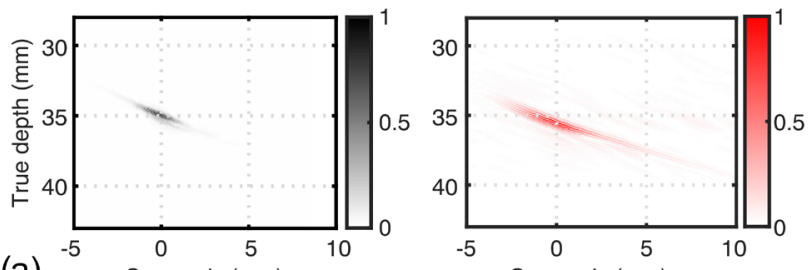

(a)

Scan axis $(\mathrm{mm})$
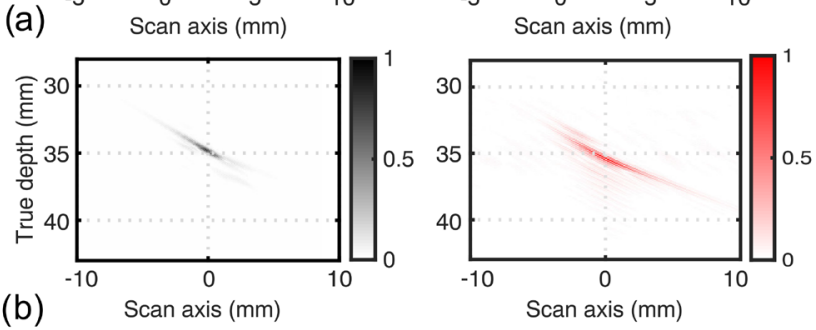

Figure 9. B-scan comparisons between simulations (grey scaled to the left) and experiments (red scaled to the right) on different surface breaking cracks in test specimen \#2 with $35 \mathrm{~mm}$ focus depth, crack height: (a) $0.5 \mathrm{~mm}$, (b) $2 \mathrm{~mm}$, (c) $5 \mathrm{~mm}$, (d) $10 \mathrm{~mm}$. 


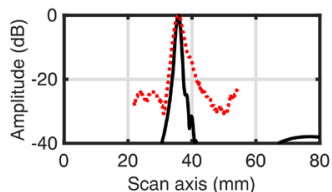

(a)

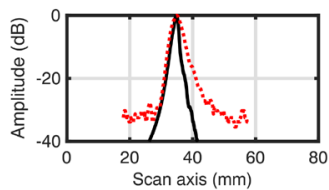

(b)

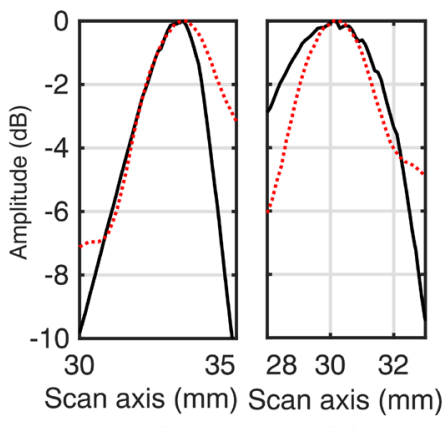

(c)

(d)

Figure 10. C-scan (echo dynamic curve) comparisons between simulations (black solid lines) and experiments (red dotted lines) on different surface breaking cracks in test specimen \#2 with $35 \mathrm{~mm}$ focus depth, crack height: (a) $0.5 \mathrm{~mm}$, (b) $2 \mathrm{~mm}$, (c) $5 \mathrm{~mm}$, (d) 10 $\mathrm{mm}$.

B-scans are incomplete and incomparable to experiments and are thus neglected in Figure 9. This also influences their C-scans in Figure 10 that only a small scan interval is comparable to experiments. It is noticed in Figure 9 that the B-scans at depth around $35 \mathrm{~mm}$ are identical while the experimental shapes have bottom part (tail) that simulations do not. These tails could be caused by beam divergence variations and possible further residual corner echos in experiments since the sound beam is focused at the backwall of the test specimen.

\subsection{Probe Skew Angle Sensitivity}

With the help of the model flexibility, a set of simulations were conducted with only the probe skew angle being varied from $-5^{\circ}$ to $5^{\circ}$ with an increment of $0.5^{\circ}$. This results in 21 simulations under the case of 45 -degree angled beam with focus depth at $50 \mathrm{~mm}$ towards an SDH at $50 \mathrm{~mm}$ depth in test specimen \#1. By comparing all these simulated A-scans with experiments, where the nominal physical probe skew angle is 0-degree, it is observed in Figure 11 that a simulated probe skew angle of \pm 3.5 gives better A-scan waveform correlation to the experiment, even if the waveform shape does not differ that much.

Figure 12 presents the maximum echo amplitudes for each simulated received signal under these probe skew angles. The values are normalized with the maximum echo amplitude obtained at probe skew angle of 0 -degree. It can be noticed that the normalized amplitude level is symmetric along 0-degree skew angle, which indicates that the waveforms in A-scans are the same for the symmetric probe skew angles.

\subsection{Sound Field Optimization}

As mentioned in the optimization process that a bandwidth of $0.02 \%$ instead of the nominal $74 \%$ is used in the simulations for optimization work, which generates sufficiently accurate maximum echo amplitude while less time-consuming. An initialized sound field optimization work is conducted towards a surface breaking crack, which has a height of $10 \mathrm{~mm}$ and tilt angle of 0 -degree that 

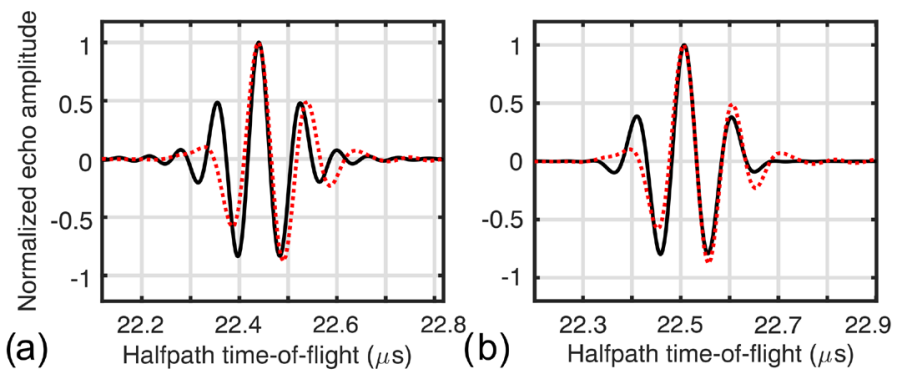

Figure 11. A-scan comparisons between simulations (black solid lines) and experiments (red dotted lines) on SDH at $50 \mathrm{~mm}$ depth in test specimen \#1 under the case of 45-degree angled beam with $50 \mathrm{~mm}$ focus depth, simulated probe skew angle of 0 -degree in (a) and \pm 3.5 -degree in (b).

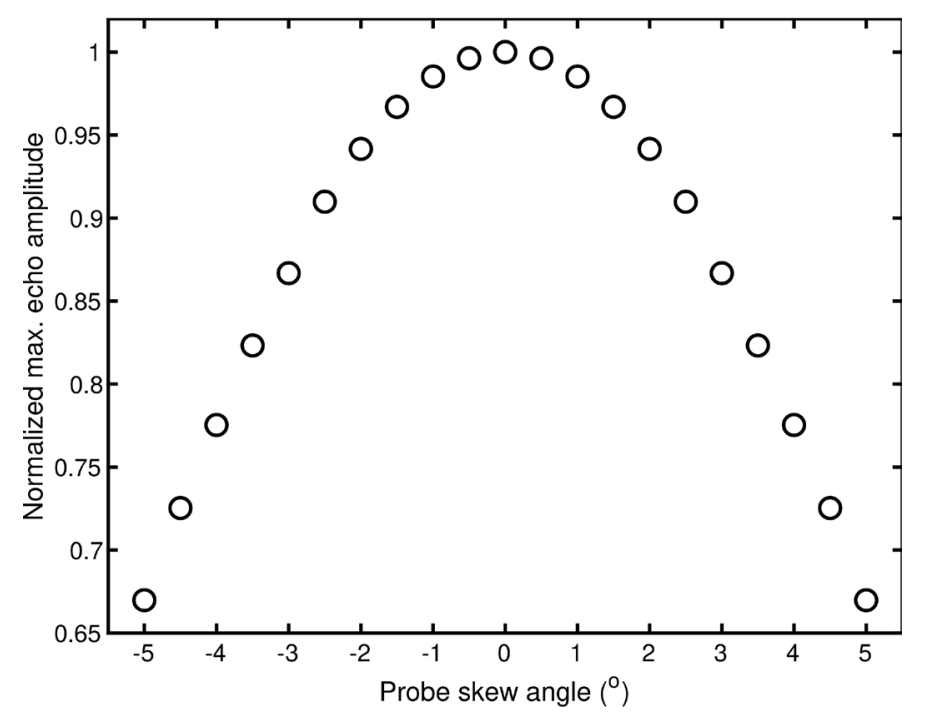

Figure 12. Maximum echo amplitude (normalized at 0-degree probe skew angle, expressed in percentage) for each simulated probe skew angle under the inspection of SDH at $50 \mathrm{~mm}$ depth in test specimen \#1 in the case of 45-degree angled beam with $50 \mathrm{~mm}$ focus depth.

corresponds to the $10 \mathrm{~mm}$ height crack on test specimen $\# 2$. The crack opening is on the bottom surface of the test specimen, so the received signal (maximum echo amplitude) is the corner echo from the crack. The decision variables are the probe (beam) angle and focusing distance. Figure 13 shows the iteration processes of the optimization for this crack. It can be seen that after around 20 iterations, a maximized (optimal) echo amplitude of around $80.2 \mathrm{~dB}$ can be found under an optimal combination of 48-degree probe angle and $45.2 \mathrm{~mm}$ focusing distance (corresponds to a focusing depth of $30.2 \mathrm{~mm}$ at this angle), i.e. the optimal solution. Comparing with the second case in Table 3, where the same crack is simulated but with an original combination of 45-degree probe angle and $49.49 \mathrm{~mm}$ focusing distance, it is obvious that the received maximum echo amplitude is larger using the optimal solution, i.e. $80.2 \mathrm{~dB}>76.9 \mathrm{~dB}$.

Noticing the third investigation case in Table 3 where the unique monochromatic frequency spectrum is simulated, the accuracy of obtained maximum echo 

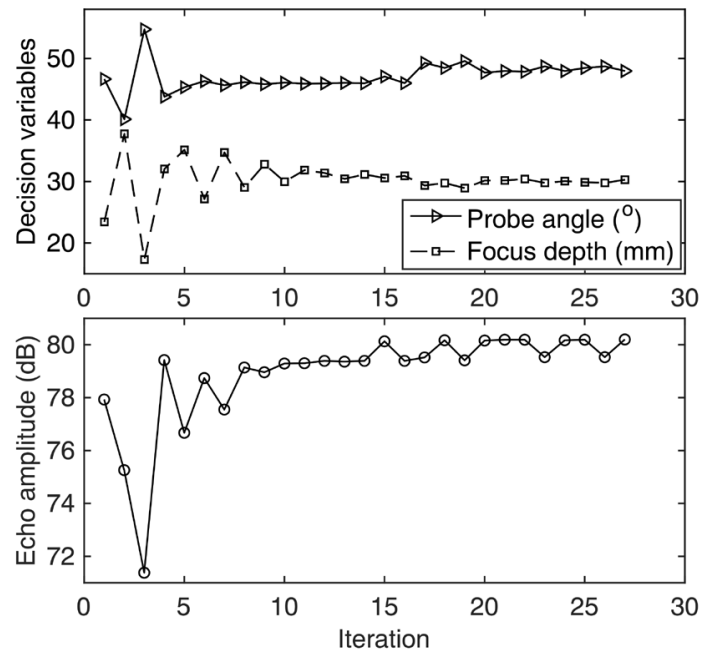

Figure 13. Optimization iterations of decision variables (probe angle and focus depth) to maximize the echo amplitude towards a surface breaking crack with height of $10 \mathrm{~mm}$ and tilt angle of 0 -degree, simulation bandwidth is $0.02 \%$.
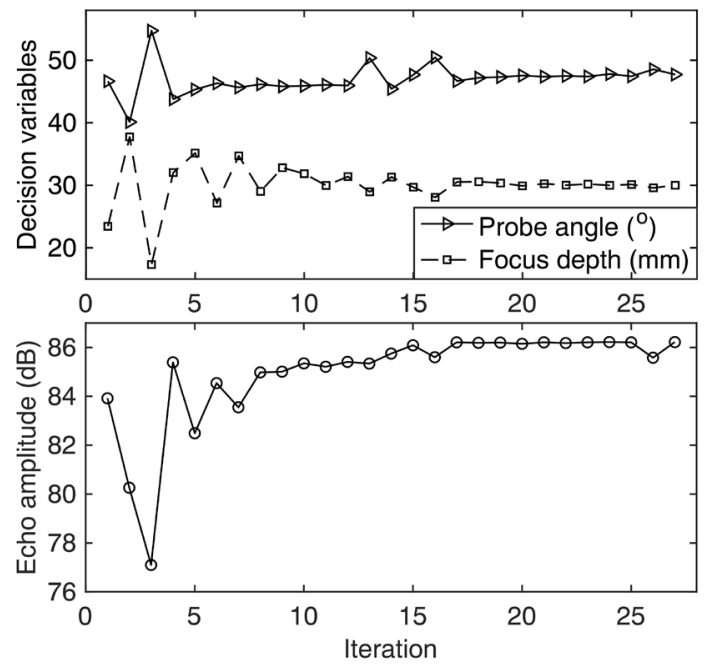

Figure 14. Optimization iterations of decision variables (probe angle and focus depth) to maximize the echo amplitude towards a surface breaking crack with height of $10 \mathrm{~mm}$ and tilt angle of 0 -degree, simulation frequency is monochromatic (bandwidth is $0 \%$ ).

amplitude cannot be guaranteed, but the simulation time is reduced significantly. Thus, an optimization trial is performed using this monochromatic frequency configuration towards the same defect, i.e. surface breaking crack with $10 \mathrm{~mm}$ height and 0-degree tilt angle. The optimization iterations can be seen in Figure 14 and the optimal combination of decision variables is found to be 48-degree probe angle and $45 \mathrm{~mm}$ focusing distance (corresponds to a focusing depth of 30 $\mathrm{mm}$ at this angle), which gives the maximized echo amplitude of $86.2 \mathrm{~dB}$. Comparing this optimal solution to the previous one using $0.02 \%$ bandwidth, it is found that the optimal combinations of the probe angle and focusing distance are almost the same whereas the maximized echo amplitudes differ. Note that it is the combination of decision variables that matters to the optimization prob- 
lem, therefore, it is reasonable to believe that a monochromatic frequency configuration can be used in the further similar optimization processes.

The increase in received signal amplitude from the surface breaking crack with $10 \mathrm{~mm}$ height and 0 -degree tilt angle by using optimal parameter set is visible in Table 4. The optimal solution using nominal 74\% bandwidth are compared with the original one (i.e. nominal bandwidth of $74 \%$ and the parameter combination of 45-degree beam angle with $35 \mathrm{~mm}$ focus depth). A $4.1 \mathrm{~dB}$ increased amplitude than original is observed. Besides, the experimental echo amplitude comparison using the original combination and the optimal one is also presented in Table 4 to verify that the optimal solution ensures an improvement of the received echo amplitude. Note that these amplitude results in the table are normalized by the one using the original combination, i.e. $76.8 \mathrm{~dB}$ with bandwidth of $74 \%$.

After the above observations, Figure 15 presents an optimization case towards a surface breaking crack with height of $10 \mathrm{~mm}$ and tilt angle of 5-degree using monochromatic frequency configuration. After around 20 iterations, the optimal combination (optimal solution) of probe angle of 56-degree and focusing distance of $46.3 \mathrm{~mm}$ (corresponds to a focusing depth of $26 \mathrm{~mm}$ at this angle) is

Table 4. Normalized comparisons of received signal amplitude using original and optimal combinations of decision variables and with different simulated bandwidth, normalization reference is $76.8 \mathrm{~dB}$.

\begin{tabular}{ccc}
\hline & Simulated amplitude delta (dB) & Experimental amplitude delta (dB) \\
\hline Original (BW74\%) & 0 & 0 \\
Optimal (BW74\%) & +4.1 & +1.4 \\
Optimal (BW0.02\%) & +3.4 & - \\
Optimal (BW0\%) & +9.4 & - \\
\hline
\end{tabular}
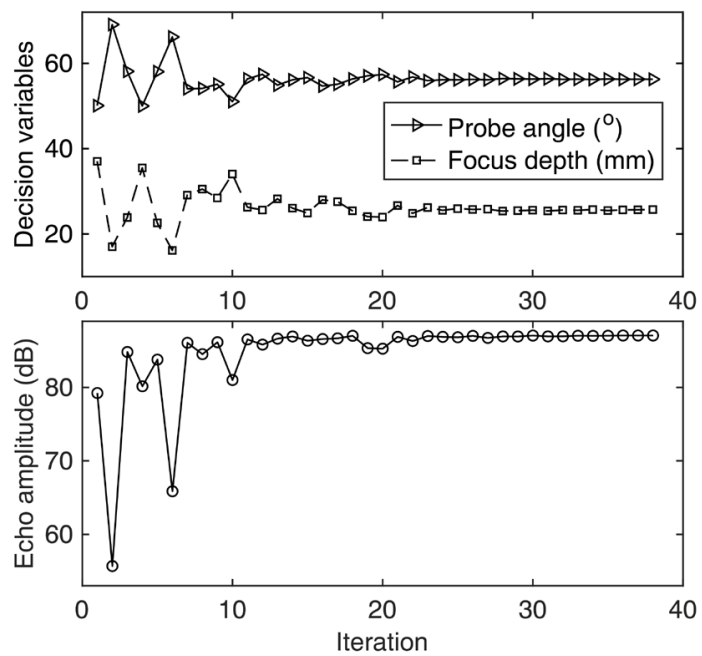

Figure 15. Optimization iterations of decision variables (probe angle and focus depth) to maximize the echo amplitude towards a surface breaking crack with height of $10 \mathrm{~mm}$ and tilt angle of 5-degree, simulation frequency is monochromatic (bandwidth is $0 \%$ ). 
found, which gives the maximized echo amplitude of $87.1 \mathrm{~dB}$. This optimal solution is also verified afterwards by using $0.02 \%$ bandwidth, which gives the maximized echo amplitude of $81.4 \mathrm{~dB}$ under probe angle of 56-degree and focusing distance of $46.9 \mathrm{~mm}$.

\section{Conclusions}

Numerical models in UT have many potential benefits to be a complement of physical experimental work and to the understanding of the techniques. To ensure the validity of these models, validation is essential, either by comparison to other validated models or to corresponding experimental scenarios. In the current work, the PA probe model included in the UT simulation software simSUNDT is further validated experimentally in terms of data presentation comparisons, i.e. A-, B- and C-scans. These data presentations under different inspection scenarios show satisfactory correlations to corresponding experiments in general, and confirm the validity of the simulation model.

After the model is validated, it is further used in exploring the optimized sound field generated by a PA probe. The sound field optimization aims at retrieving the maximized echo amplitude as a function of the defect characteristics (size and tilt angle) by adjusting a combination of decision variables, i.e. probe angle and focusing distance, of PA probe. A surface breaking crack with certain height and tilt angle is considered as the targeted defect. It is observed before the optimization work that, instead of using the nominal probe parameter (i.e. bandwidth), a change of this parameter not only maintains the echo amplitude level, but also significantly reduces the simulation time. Thus, this is used as an approach in the optimization process. It is then found by comparing this optimization result with the one obtained using monochromatic frequency configuration that, the latter configuration ends up with the same optimal combination of decision variables as the first one, even if the obtained maximized echo amplitudes differ. In other words, the monochromatic configuration can reduce the simulation time significantly while still results in a set of reasonable optimal decision variables. A brief experimental verification of the optimal solution is presented accordingly and the improvement of sound field, i.e. the received signal amplitude in current situation, using the optimal solution is clearly observed. Thus, this optimization scheme can be used in optimization work with other defect characteristics.

\section{Acknowledgements}

This work was supported by the Swedish innovation agency VINNOVA under Grant 2017-04856. It is performed in collaboration with GKN Aerospace Engine System and Chalmers University of Technology. The national aeronautical research program (NFFP7) is greatly acknowledged.

\section{Conflicts of Interest}

The authors declare no conflicts of interest regarding the publication of this paper. 


\section{References}

[1] Hagemaier, D. (1974) SAE Transactions, 83, 2767-2796. https://doi.org/10.4271/740811

[2] Drinkwater, B.W. and Wilcox, P.D. (2006) NDT \& E International, 39, 525-541. https://doi.org/10.1016/j.ndteint.2006.03.006

[3] Holmer, G., Daniels, W. and Zettervall, T. (2017) Evaluation of the Simulation Software CIVA for Qualification Purpose.

https://www.stralsakerhetsmyndigheten.se/publikationer/rapporter/sakerhet-vid-ka rnkraftverken/2017/201729

[4] Lopez, A.B., Santos, J., Sousa, J.P., Santos, T.G. and Quintino, L. (2019) Journal of Nondestructive Evaluation, 38, 1-11. https://doi.org/10.1007/s10921-019-0600-y

[5] Chatillon, S., Robert, S., Calmon, P., Daniel, G., Cartier, F., et al. (2015) AIP Conference Proceedings, 1650, 1847-1855. https://doi.org/10.1063/1.4914810

[6] Lei, X., Wirdelius, H. and Rosell, A. (2020) Ultrasonics, 108, Article ID: 106217. https://doi.org/10.1016/j.ultras.2020.106217

[7] Jansson, P.A. (2000) AIP Conference Proceedings, 509, 57-64. https://doi.org/10.1063/1.1306034

[8] Boström, A. and Eriksson, A.S. (1993) Proceedings of the Royal Society of London. Series A: Mathematical and Physical Sciences, 443, 183-201. https://doi.org/10.1098/rspa.1993.0139

[9] Bövik, P. and Boström, A. (1997) The Journal of the Acoustical Society of America, 102, 2723-2733. https://doi.org/10.1121/1.420326

[10] Boström, A. and Wirdelius, H. (1995) The Journal of the Acoustical Society of America, 97, 2836-2848. https://doi.org/10.1121/1.411850

[11] Wirdelius, H. (2007) Experimental validation of the UTDefect simulation software. Proceedings of 6 th International Conference on NDE in Relation to Structural Integrity for Nuclear and Pressurized Components, Budapest, 8-10 October 2007.

[12] Boström, A. and Bövik, P. (2003) International Journal of Solids and Structures, 40, 3493-3505. https://doi.org/10.1016/S0020-7683(03)00150-1

[13] Auld, B.A. (1979) Wave Motion, 1, 3-10. https://doi.org/10.1016/0165-2125(79)90020-9

[14] Grešovnik, I. (2007) Simplex Algorithms for Nonlinear Constraint Optimization Problems.

[15] Poles, S. (2003) The Simplex Method. 